\author{
Chien-Ching Ma \\ Associate Professor \\ Department of Mechanical Engineering, \\ National Taiwan University, \\ Taipei, Taiwan 10764
}

\section{Dynamic Mixed Mode I-II Crack Kinking Under Oblique Stress Wave Loading in Brittle Solids}

The dynamic stress intensity factors of an initially stationary semi-infinite crack in an unbounded linear elastic solid which kinks at some time $\mathrm{t}_{\S}$ after the arrival of a stress wave is obtained as a function of kinking crack tip velocity $\mathrm{v}$, kinking angle $\delta$, incident stress wave angle $\alpha$, time $\mathrm{t}$, and the delay time $\mathrm{t}_{\mathrm{f}}$. A perturbation method, using the kinking angle $\delta$ as the perturbation parameter, is used. The method relies on solving simple problems which can be used with linear superposition to solve the problem of a kinked crack. The solutions can be compared with numerical results and other approximate results for the case of $\mathrm{t}_{\mathrm{f}}=0$ and give excellent agreement for a large range of kinking angles. The elastodynamic stress intensity factors of the kinking crack tip are used to compute the corresponding fluxes of energy into the propagating crack-tip, and these results are discussed in terms of an assumed fracture criterion.

\section{Introduction}

When dynamic loading is applied to a body with an internal crack, the stress gradually intensifies at the crack-tip and after some finite delay time, the resulting stress waves may cause the initiation of crack growth and continued crack propagation. A frequently observed fracture event is the kinking or bifurcation of an initially straight crack. The direction of propagation, as well as the velocity of crack propagation, will depend on the local stress field around the crack-tip. To understand the observed bifurcation events in brittle material, the dynamic solution for cracks which suddenly branch or kink is required. The problem of a crack that is branching at an arbitrary angle with the primary crack is difficult to solve, and much of the analytical work is elastostatic in nature. A great deal of progress has been made recently in analyzing the problem of elastodynamic crack branching problems in homogeneous, isotropic elastic solids. One method which seems to have potential for analytic solutions was proposed by Achenbach and Varatharajulu (1974). The method takes advantage of the self-similarity of the particle velocity of the diffracted field, which occurs when appropriate conditions on the incident wave and the crack geometry are satisfied. This method was used by Burgers and Dempsey (1982) to construct exact solutions of symmetric crack bifurcation in antiplane strain for a specific angle. Subsequently, Dempsey, Kuo, and Achenbach

Contributed by the Applied Mechanics Division of ThE AMERICAN SocietY of Mechanical Engineers for publication in the Journal of Applied MECHANICS.

Discussion on this paper should be addressed to the Technical Editor, Leon M. Keer, The Technical Institute, Northwestern University, Evanston, IL 60201, and will be accepted until two months after final publication of the paper itself in the Journal of Appied Mechanics. Manuscript received by the ASME Applied Mechanics Division, May 2, 1988; final revision, April 1, 1989.
(1982) have used a conformal mapping to obtain the analytical solution for the mode III crack kinking problem for stress wave loading. The problem of symmetric and asymmetric crack bifurcation in mode III has been studied by Dempsey, Kuo, and Bentley (1986). The solutions in Burgers and Dempsey (1982) and Dempsey, Kuo and Achenbach (1982) verified the numerical method being applied by Burgers (1982). This method has since been used by Burgers (1983) and Burgers and Dempsey (1984) to provide numerical solutions to the plane-strain crack kinking and bifurcation problems, respectively.

For an important range of kinking angles, the elastodynamic crack kinking stress intensity factors are affected more by the loading of the new crack faces than by the wedge geometry. This suggests that a suitable first-order approximation would be to ignore the wedge geometry and to compute the elastodynamic stress intensity factors by considering a crack propagating in its own plane provided, however, that the new faces are subjected to traction corresponding to those of the branched crack. This approximate method for both mode III and mixed mode I-II crack kinking under stress wave loading was investigated by Achenbach, Kuo, and Dempsey (1984). In all the results mentioned, the problems are restricted to being selfsimilar; it is assumed that the new crack initiates out of the original crack-tip at an angle at the same time as the stress wave loading arrived at the crack-tip.

It was observed by Achenbach (1970) that if a plane-stress pulse strikes a half-plane crack in an initially undisturbed medium, instantaneous crack propagation can occur only if the stress pulse front carries a square-root singular stress. Hence, it will greatly improve the model by allowing a finite delay time in the initiation of the nonplanar crack. This finite delay time effect also observed in a series of paper by Ravi-Chandar and Knauss (1984a, b, c, d). But if we do so, the problem loses 
its self-similar nature. The only solutions for the newly initiated crack propagating after a delay time have been first obtained by Freund $(1973,1974)$, and these solutions are restricted to the crack remaining straight. More recently, a finite delay time has been included in the initiation of the nonplanar crack by Ma and Burgers (1986), in which the approximate method in Achenbach, Kuo, and Dempsey (1984) has been used for analyzing the antiplane-strain case. Ma and Burgers (1987) extended the delay time effect to the in-plane case for incident stress wave which is parallel to the crack faces.

The analysis undertaken here is the extension of the previous work in which both the incident longitudinal and transverse stress wave parallel to the crack faces was solved. We consider the dynamic crack growth out of the original semi-infinite crack at an angle to the original crack at some time after the oblique longitudinal (or transverse) stress-wave loading initially interacts with the crack tip. The geometry for the kinked crack under consideration with the wavefront pattern for stress-wave loading is shown in Fig. 1. A perturbation method is used to obtain the first-order solution of the dynamic stress intensity factor near the kinking crack-tip. When the kinking angle is zero, the solutions obtained in this paper reduce to the results of Freund $(1973,1974)$, and reduce to the solutions of Achenbach, Kuo, and Dempsey (1984) as the delay time tends to zero. The energy flux into the propagating kinked crack-tip can be obtained from the dynamic stress intensity factors, and those results are discussed in terms of an assumed fracture criterion.

\section{Required Fundamental Solutions}

Consider a stress-free linear elastic homogeneous isotropic infinite medium that contains a stationary semi-infinite crack, which will be referred to as the original crack, which lies along the negative $x$-axis with the origin of the coordinate system at the crack-tip. At time $t=0$, a horizontally-polarized longitudinal wave (or transverse wave) strikes the stationary cracktip at angle of incidence $\alpha$. A short time later, at $t=t_{f}$, a crack referred to as the new crack propagates out of the tip

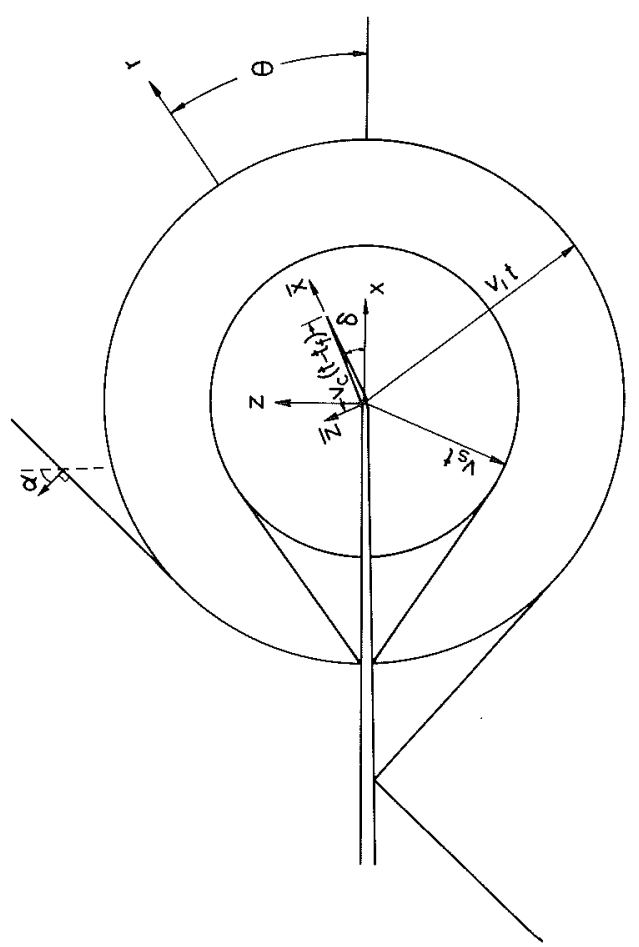

Fig. 1 Stress wave front pattern for a planar stress-wave impacting a kinking crack of the semi-infinite crack with a constant velocity $v$ (less than the Rayleigh wave speed) making an angle $\delta$ with the original crack, thus producing a kinked crack.

The field solution for a kinked-crack geometry can be considered as the superposition of the field generated by diffraction of the incident wave by the stationary crack and the field from the new crack faces subjected to crack-face tractions, which are opposite in sign to the stresses computed from the stationary crack problem. The fields generated by kinking of a semi-infinite crack upon diffraction of a longitudinal or transverse stress wave are extremely difficult to analyze. The analysis involves coupled integral equations, which must be solved numerically. (See Burgers (1983) and Burgers and Dempsey (1984).) We use the first-order approximation of the dynamic stress intensity factor for a kinked crack, which can be expressed by the stress intensity factor for a straight crack, propagating in its own plane, subjected to the negative of the traction computed from the stationary crack problem along the line of the kinked crack. The solutions for the approximation method for the kinking crack under stress-wave loading can be separated into a number of different problems, all of which are relevant problems in their own right. By building up the so-called fundamental solutions of more basic problems, the solution of the final problem can then be solved. Some fundamental solutions needed for solving the dynamic stress intensity factor of the kinking crack will be presented.

In a stationary coordinate $x-z$ system, a homogeneous, isotropic, linearly elastic medium is governed by the two-dimensional wave equations

$$
\begin{aligned}
& \nabla^{2} \phi-a^{2} \ddot{\phi}=0, \\
& \nabla^{2} \psi-b^{2} \ddot{\psi}=0,
\end{aligned}
$$

where

$$
a=\sqrt{\frac{\rho}{\lambda+2 \mu}}=\frac{1}{v_{l}}, b=\sqrt{\frac{\rho}{\mu}}=\frac{1}{v_{s}} .
$$

$a$ and $b$ are the slowness of longitudinal and shear waves, respectively, $\mu$ and $\rho$ are the shear modulus and the mass density of the material, and $\lambda$ is the Lamé elastic constant. The displacements are derived from the potentials according to $u=$ $\phi_{,_{x}}-\psi_{, z}, w=\phi,_{z}+\psi_{, x}$, where $u$ and $w$ are the displacements in the $x$ and $z$ directions. The stresses can be written in terms of the potentials by means of Hooke's law.

Diffraction by a Stationary Crack. Consider a special problem for a tensile stress loading applied uniformly on the stationary crack faces $z=0$. The following mixed boundary conditions on $z=0$ are considered:

$$
\begin{aligned}
& \sigma_{z z}^{I}(x, 0, t)=-\sigma_{o} H\left(t+x a^{*}\right) \text { for }-\infty<x<0, \\
& \sigma_{x z}^{I}(x, 0, t)=0 \text { for }-\infty<x<\infty, \\
& w(x, 0, t)=0 \text { for } 0<x<\infty .
\end{aligned}
$$

where $a^{*}=a \sin \alpha$ and $H$ represents the unit step function. The full field solutions of stresses for $-\pi / 2<\theta<\pi / 2$ are

$$
\begin{aligned}
\frac{\sigma_{z z}^{I}}{A}=- & \int_{a r}^{t} \operatorname{Im}\left[\frac{\left(2 \lambda^{2}-b^{2}\right)^{2} \Phi(s)}{(a+\lambda)^{1 / 2}}\right]_{\lambda=\lambda_{L}} d s \\
& -\int_{b r}^{t} \operatorname{Im}\left[4 \lambda^{2}(a-\lambda)^{1 / 2}\left(b^{2}-\lambda^{2}\right)^{1 / 2} \Phi(s)\right]_{\lambda=\lambda_{T}} d s, \\
\frac{\sigma_{x z}^{I}}{A}=- & \int_{a r}^{t} \operatorname{Im}\left[2 \lambda\left(2 \lambda^{2}-b^{2}\right)(a-\lambda)^{1 / 2} \Phi(s)\right]_{\lambda=\lambda_{L}} d s \\
& +\int_{b r}^{t} \operatorname{Im}\left[2 \lambda\left(2 \lambda^{2}-b^{2}\right)(a-\lambda)^{1 / 2} \Phi(s)\right]_{\lambda=\lambda_{T}} d s,
\end{aligned}
$$




$$
\begin{aligned}
\frac{\sigma_{x x}^{I}}{A}=\int_{a r}^{t} & \operatorname{Im}\left[\frac{\left(2 \lambda^{2}-b^{2}\right)\left(2 \lambda^{2}+b^{2}-2 a^{2}\right) \Phi}{(a+\lambda)^{1 / 2}}\right]_{\lambda=\lambda_{L}} d s \\
& +\int_{b r}^{t} \operatorname{Im}\left[4 \lambda^{2}(a-\lambda)^{1 / 2}\left(b^{2}-\lambda^{2}\right)^{1 / 2} \Phi\right]_{\lambda=\lambda_{T}} d s,
\end{aligned}
$$

where

$$
\begin{gathered}
A=\frac{\sigma_{o} \omega_{+}^{o}\left(a^{*}\right)}{\pi k}, k=2\left(b^{2}-a^{2}\right), \\
\omega_{+}^{o}(\lambda)=\frac{(a+\lambda)^{1 / 2}}{(c+\lambda) S_{+}^{o}(\lambda)}, \\
\lambda_{L}(s)=-\frac{s}{r} \cos \theta+i\left(\frac{s^{2}}{r^{2}}-a^{2}\right)^{1 / 2} \sin |\theta|, \\
\lambda_{T}(s)=-\frac{s}{r} \cos \theta+i\left(\frac{s^{2}}{r^{2}}-b^{2}\right)^{1 / 2} \sin |\theta|,
\end{gathered}
$$

$$
\begin{gathered}
S_{ \pm}^{o}(\lambda)= \\
\exp \left(-\frac{1}{\pi} \int_{a}^{b} \tan ^{-1}\left[\frac{4 y^{2}\left(y^{2}-a^{2}\right)^{1 / 2}\left(b^{2}-y^{2}\right)^{1 / 2}}{\left(b^{2}-2 y^{2}\right)^{2}}\right] \frac{d y}{y \pm \lambda}\right), \\
\Phi(s)=\frac{\partial \lambda / \partial s}{\left(\lambda-a^{*}\right)(\lambda-c) S_{-}^{o}(\lambda)} .
\end{gathered}
$$

$c=1 / v_{R}$ is the slowness of the Rayleigh wave and satisfies the equation

$$
\left(2 c^{2}-b^{2}\right)^{2}+4 c^{2}\left(a^{2}-c^{2}\right)^{1 / 2}\left(b^{2}-c^{2}\right)^{1 / 2}=0 .
$$

The first two terms of the asymptotic expansion as $r \rightarrow 0$ of the stresses are

$$
\begin{gathered}
\sigma_{z z}^{I} \approx \frac{2}{\pi} \sigma_{o} \omega_{+}^{o}\left(a^{*}\right)\left(\frac{t}{r}\right)^{1 / 2} \cos \frac{\theta}{2}\left[1+\sin \frac{\theta}{2} \sin \frac{3 \theta}{2}\right]-\sigma_{o}+o(1), \\
\sigma_{x z}^{I} \approx \frac{2}{\pi} \sigma_{o} \omega_{+}^{o}\left(a^{*}\right)\left(\frac{t}{r}\right)^{1 / 2} \cos \frac{\theta}{2} \sin \frac{\theta}{2} \cos \frac{3 \theta}{2}+o(1), \\
\sigma_{x x}^{I} \approx \frac{2}{\pi} \sigma_{o} \omega_{+}^{o}\left(a^{*}\right)\left(\frac{t}{r}\right)^{1 / 2} \cos \frac{\theta}{2}\left[1-\sin \frac{\theta}{2} \sin \frac{3 \theta}{2}\right]-\sigma_{o} C_{x x}+o(1),
\end{gathered}
$$

where

$$
C_{x x}=\frac{b^{2}-2 a^{2}}{b^{2}}+\frac{\sqrt{a}\left(2 c^{2}-b^{2}\right)}{c^{2} S_{+}^{o}(0) S_{-}^{o}(c)(a+c)} .
$$

The second term in (5), which is $O(1)$ in the asymptotic expansion, will play a significant role in the crack kinking analysis.

For the shear loading applied uniformly on the crack faces, the deformation will occur in mode II. The mixed boundary conditions are then

$$
\begin{aligned}
& \sigma_{z z}^{I I}(x, 0, t)=0 \text { for }-\infty<x<\infty, \\
& \sigma_{x z}^{I I}(x, 0, t)=-\sigma_{o} H\left(t+x a^{*}\right) \text { for }-\infty<x<0, \\
& u^{*}(x, 0, t)=0 \text { for } 0<x<\infty,
\end{aligned}
$$

where $u^{*}$ is the component of displacement in the $x$-direction. The full-field solutions for stresses evaluated for $-\pi / 2<\theta$ $<\pi / 2$ are

$$
\begin{aligned}
\frac{\sigma_{z z}^{I I}}{B}=\int_{a r}^{t} & \operatorname{Im}\left[2 \lambda\left(b^{2}-2 \lambda^{2}\right)(b-\lambda)^{1 / 2} \Phi(s)\right]_{\lambda=\lambda_{L}} d s \\
& -\int_{b r}^{t} \operatorname{Im}\left[2 \lambda\left(b^{2}-2 \lambda^{2}\right)(b-\lambda)^{1 / 2} \Phi(s)\right]_{\lambda=\lambda_{T}} d s
\end{aligned}
$$

$$
\begin{aligned}
\frac{\sigma_{x z}^{I I}}{B}=-\int_{a r}^{t} \operatorname{Im}\left[4 \lambda^{2}(b-\lambda)^{1 / 2}\left(a^{2}-\lambda^{2}\right)^{1 / 2} \Phi(s)\right]_{\lambda=\lambda_{L}} d s \\
-\int_{b r}^{t} \operatorname{Im}\left[\frac{\left(2 \lambda^{2}-b^{2}\right)^{2} \Phi(s)}{(b+\lambda)^{1 / 2}}\right]_{\lambda=\lambda_{T}} d s,
\end{aligned}
$$

$$
\begin{aligned}
\frac{\sigma_{x x}^{I I}}{B}=\int_{a r}^{t} \operatorname{Im}\left[2 \lambda(b-\lambda)^{1 / 2}\left(2 \lambda^{2}+b^{2}-2 a^{2}\right) \Phi\right]_{\lambda=\lambda_{L}} d s \\
+\int_{b r}^{t} \operatorname{Im}\left[2 \lambda(b-\lambda)^{1 / 2}\left(b^{2}-2 \lambda^{2}\right) \Phi\right]_{\lambda=\lambda_{T}} d s
\end{aligned}
$$

where

$$
B=\frac{\sigma_{o} u_{+}^{o}\left(a^{*}\right)}{\pi k}, u_{+}^{o}(\lambda)=\frac{(b+\lambda)^{1 / 2}}{(c+\lambda) S_{+}^{o}(\lambda)} .
$$

For $r \rightarrow 0$, the leading two terms of the asymptotic expansion of (7) are

$$
\begin{gathered}
\sigma_{z z}^{I I} \approx \frac{2}{\pi} \sigma_{o} u_{+}^{o}\left(a^{*}\right)\left(\frac{t}{r}\right)^{1 / 2} \cos \frac{\theta}{2} \sin \frac{\theta}{2} \cos \frac{3 \theta}{2}+o(1), \\
\sigma_{x z}^{I I} \approx \frac{2}{\pi} \sigma_{o} u_{+}^{o}\left(a^{*}\right)\left(\frac{t}{r}\right)^{1 / 2} \cos \frac{\theta}{2}\left[1-\sin \frac{\theta}{2} \sin \frac{3 \theta}{2}\right]-\sigma_{o}+o(1), \\
\sigma_{x x}^{I I} \approx-\frac{2}{\pi} \sigma_{o} u_{+}^{o}\left(a^{*}\right)\left(\frac{t}{r}\right)^{1 / 2} \sin \frac{\theta}{2}\left[2+\cos \frac{\theta}{2} \cos \frac{3 \theta}{2}\right]+o(1)
\end{gathered}
$$

Point Moving Loads of Growing Crack. Consider a cracktip which is at rest at $x=0$, and suppose there are no loads acting on the body for $t<0$. At time $t=0$, the crack-tip begins to move in the $x$ direction at speed $v$ and, simultaneously, a symmetric pair of concentrated normal forces appears at the crack-tip. For $t>0$, the concentrated forces increase linearly in time and begin to move in the $x$-direction with speed $u<v$. The boundary conditions are

$$
\begin{aligned}
\left(\sigma_{z z}^{F}\right)_{1}(x, 0, t) & =(m t+n) \Delta(x-u t) H(t) \text { for }-\infty<x<v t, \\
\left(\sigma_{x z}^{F}\right)_{1}(x, 0, t) & =0 \quad \text { for }-\infty<x<\infty, \\
w_{1}(x, 0, t) & =0 \quad \text { for } \quad v t<x<\infty,
\end{aligned}
$$

where $\Delta$ is the Dirac delta function and $m$ and $n$ are arbitrary parameters. The stress intensity factor of this problem was obtained by Freund (1973)

$$
K_{I}^{F}=\frac{2 m h^{2} \omega_{+}^{\prime} \sqrt{2 t}}{\sqrt{\pi}(1-a / d)^{1 / 2}}-\frac{\sqrt{2} n h \omega_{+}(h)}{(1-a / d)^{1 / 2} \sqrt{\pi t}},
$$

where

$$
\begin{gathered}
\omega_{+}^{\prime}(h)=\frac{\partial \omega_{+}(h)}{\partial h}, \omega_{+}(\lambda)=\frac{\alpha_{+}(\lambda)}{\left(\lambda+c_{2}\right) S_{+}(\lambda)} \\
S_{ \pm}(\lambda)=\exp \left(-\frac{1}{\pi} \int_{a_{2,1}}^{b_{2,1}}\right. \\
\left.\times \tan ^{-1}\left[\frac{4 \eta^{2}|\alpha||\beta|}{\left(2 \eta^{2}-b^{2}-b^{2} \eta^{2} / d^{2} \mp 2 b^{2} \eta / d\right)^{2}}\right] \frac{d \eta}{\eta \pm \lambda}\right), \\
\alpha(\lambda)=\left(a^{2}-\lambda^{2}+a^{2} \lambda^{2} / d^{2}-2 a^{2} \lambda / d\right)^{1 / 2} \\
\beta(\lambda)=\left(b^{2}-\lambda^{2}+b^{2} \lambda^{2} / d^{2}-2 b^{2} \lambda / d\right)^{1 / 2} \\
\alpha_{ \pm}=[a \pm \lambda(1 \mp a / d)]^{1 / 2}, \\
a_{2}=\frac{a}{1-a / d}, b_{2}=\frac{b}{1-b / d}, c_{2}=\frac{c}{1-c / d}
\end{gathered}
$$

$d=1 / v$ is the slowness of the crack velocity. The parameter $h=1 /(v-u)$ is the inverse of the relative speed between the moving load and the crack-tip. The other fundamental solution 
needed is that produced by concentrated shear forces appearing at the crack-tip and then moving in the $x$ direction with speed $u$. The boundary conditions are

$$
\begin{aligned}
\left(\sigma_{z z}^{F}\right)_{2}(x, 0, t) & =0 \text { for }-\infty<x<\infty, \\
\left(\sigma_{x z}^{F}\right)_{2}(x, 0, t) & =(m t+n) \Delta(x-u t) H(t) \text { for }-\infty<x<v t, \\
u^{*}(x, 0, t) & =0 \text { for } v t<x<\infty .
\end{aligned}
$$

The stress intensity factor for this case is

$$
K_{I I}^{F}=\frac{2 m h^{2} u_{+}^{\prime} \sqrt{2 t}}{\sqrt{\pi}(1-b / d)^{1 / 2}}-\frac{\sqrt{2} n h u_{+}(h)}{(1-b / d)^{1 / 2} \sqrt{\pi t}},
$$

where

$$
\begin{gathered}
u_{+}(\lambda)=\frac{\beta_{+}(\lambda)}{\left(\lambda+c_{2}\right) S_{+}(\lambda)}, \\
\beta_{ \pm}(\lambda)=[b \pm \lambda(1 \mp b / d)]^{1 / 2} .
\end{gathered}
$$

With these fundamental solutions at hand, we are now able to construct the solution of the stress intensity factor for the kinking crack.

\section{Crack Kinking Due to an Incident Longitudinal Wave}

Consider the incident longitudinal step-stress tensile wave of the form

$$
\sigma_{\bar{z} \bar{z}}^{i}=\sigma_{0} H(t+a r \sin (\alpha-\theta)) .
$$

The stresses of the stationary crack problem can be obtained by superimposing the solutions of the symmetric and antisymmetric problems proposed in the previous section. Relative to polar coordinates,

$$
\sigma_{\theta \theta}^{s}=\sigma_{\theta \theta}^{i}+\sigma_{\theta \theta}^{d}, \sigma_{\theta r}^{s}=\sigma_{\theta r}^{i}+\sigma_{\theta r}^{d},
$$

where

$$
\begin{gathered}
\sigma_{\theta \theta}^{i}=\sigma_{o}\left[1-2\left(\frac{a}{b}\right)^{2} \sin ^{2}(\alpha-\theta)\right], \\
\sigma_{\theta r}^{i}=-\sigma_{o}\left(\frac{a}{b}\right)^{2} \sin 2(\alpha-\theta),
\end{gathered}
$$

$\sigma_{\theta \theta}^{d}=\frac{1}{2}(1-\cos 2 \theta) \sigma_{x x}^{L}+\frac{1}{2}(1+\cos 2 \theta) \sigma_{z z}^{L}-\sin 2|\theta| \sigma_{x z}^{L}$,

$\sigma_{\theta r}^{d}=\left[-\frac{1}{2} \sin 2|\theta| \sigma_{x x}^{L}+\frac{1}{2} \sin 2|\theta| \sigma_{z z}^{L}+\cos 2 \theta \sigma_{x z}^{L}\right] \operatorname{sgn}(\theta)$

and

$$
\begin{gathered}
\sigma_{i j}^{L}=\left[1-2\left(\frac{a}{b}\right)^{2} \sin ^{2} \alpha\right] \sigma_{i j}^{I}-\left(\frac{a}{b}\right)^{2} \sin 2 \alpha \sigma_{i j}^{I I} \operatorname{sgn}(\theta) \\
i j=x x, z z, x z,
\end{gathered}
$$

where $\sigma_{\theta \theta}^{i}$ represents the incident field and $\sigma_{\theta \theta}^{d}$ is the diffraction field. $\sigma_{x x}^{I}, \sigma_{x z}^{I}$, and $\sigma_{z z}^{I}$ are given in (4). The first-order approximation of the dynamic stress intensity factor for a kinked crack with delay time $t_{f}$ can be expressed by the stress intensity factor for a straight crack propagating in its own plane subjected to the negative of the traction given in (14) on the new crack faces. This approximation method is discussed more fully by Achenbach and Kuo (1985). The appropriate boundary conditions are:

(1) for the mode I stress intensity factor

$$
\begin{aligned}
& \sigma_{\bar{z} \bar{z}}=0 \text { for } \bar{x}<0, \\
& \sigma_{\bar{z} \bar{z}}=-\sigma_{\theta \theta}^{s}(\bar{x} / t, \theta=\delta) \text { for } 0<\bar{x}<v\left(t-t_{f}\right)
\end{aligned}
$$

and

(2) for the mode II stress intensity factor

$$
\begin{aligned}
& \sigma_{\bar{z} \bar{x}}=0 \text { for } \bar{x}<0, \\
& \sigma_{\bar{z} \bar{x}}=-\sigma_{\theta r}^{s}(\bar{x} / t, \theta=\delta) \text { for } 0<\bar{x}<v\left(t-t_{f}\right),
\end{aligned}
$$

where the $\bar{x}$-axis lies along the kinked crack line and $\delta$ is the kinked angle as shown in Fig. 1.

Mode I Stress Intensity Factor. The stress intensity factor of the kinking crack due to the loading from the diffraction part of the stationary crack field can be constructed from the fundamental solution of equation (10) by choosing $m=-1$, $n=-t^{*}=-h t_{f} / d$, replacing $t$ by $t-t^{*}$, and integrating over the appropriate range of $u=\bar{x} / t$. The mode I stress intensity factor for the propagating kinked crack is

$$
\begin{aligned}
\left(K_{I}^{L}\right)_{d}(t, v, \delta)= & \int_{0}^{v\left(t-t_{f}\right) / t} K_{I}^{F}\left(m=-1, n=-t^{*}, t\right. \\
& \left.-t^{*}\right) \sigma_{\theta \theta}^{d}\left(\frac{1}{u}, \delta\right) d u \\
= & -\int_{d}^{d^{*}} 2\left[\frac{2 t_{f}}{\pi d(1-a / d)}\right]^{1 / 2}\left[\omega _ { + } ( h ) \left(d^{*}\right.\right. \\
& \left.-h)^{1 / 2}\right]_{h}^{\sigma_{\theta \theta}^{d}\left(\frac{h}{v h-1}, \delta\right) d h}
\end{aligned}
$$

where

$$
d^{*}=\frac{t}{v t_{f}}
$$

The integral in (21) is suited to integration by parts. It gives a more tractable form than (21) and allows us to get very simple closed-form results in some special cases. By careful analysis, we find that the function $\sigma_{\theta \theta}^{d}$ has a square-root singularity at $h=d$. Hence, if integration by parts is applied, neither the integrated term nor the remaining integral will exist, even though the sum exists. To get around this difficulty, the method suggested by Freund (1973) is applied. The lower limit of integration in (21) is replaced by $d+\epsilon, \epsilon<<d$. It can be shown that those terms which are singular at $\epsilon=0$ exactly cancel each other, and the desired result can be obtained by taking the limit as $\epsilon \rightarrow 0$.

Integration by parts of (21) and making use of the explicit expression for $\sigma_{\theta \theta}^{d}$ yields

$$
\begin{aligned}
&\left(K_{I}^{L}\right)_{d}(t, v, \delta)=\lim _{\epsilon \rightarrow 0} 2\left[\frac{2 t_{f}}{\pi d(1-a / d)}\right]^{1 / 2}\left\{\sigma _ { o } \omega _ { + } ( d ) \left(d^{*}\right.\right. \\
&-d)^{1 / 2}\left[\frac{2 d \Gamma_{L}}{\pi \epsilon^{1 / 2}\left(c+a^{*}\right) S_{+}^{o}\left(a^{*}\right)}-\Sigma_{L}\right]+ \\
&\left.+\int_{d+\epsilon}^{d^{*}} \omega_{+}(h)\left(d^{*}-h\right)^{1 / 2}\left(\sigma_{\theta \theta}^{d}\right)_{h} d h\right\}+o(1) \\
&= 2 \sigma_{o}\left[\frac{2 t_{f}}{\pi d(1-a / d)}\right]^{1 / 2}\left\{-\omega_{+}(d)\left(d^{*}-d\right)^{1 / 2} \Sigma_{L}\right. \\
&\left.+\frac{d \omega_{+}(d) \Gamma_{L}}{\left(c+a^{*}\right) S_{+}^{o}\left(a^{*}\right)}+\frac{d}{\pi\left(c+a^{*}\right) S_{+}^{o}\left(a^{*}\right)}\right]_{d}^{d^{*}} \frac{\left(d^{*}-h\right)^{1 / 2}}{(h-d)^{1 / 2}} \\
&\left.\times\left[\frac{\omega_{+}(d) \Gamma_{L}}{(h-d)}-\frac{\omega_{+}(h) d^{5 / 2}}{k(h-d)^{3}}\left(\sigma_{\theta \theta}^{L}\right)_{h}^{*}\right] d h\right\}+o(1)
\end{aligned}
$$

where

$$
\begin{aligned}
\Gamma_{L}=[1 & \left.-2\left(\frac{a}{b}\right)^{2} \sin ^{2} \alpha\right] \cos ^{3} \frac{\delta}{2}\left(a+a^{*}\right)^{1 / 2} \\
& +\frac{3}{4}\left(\frac{a}{b}\right)^{2} \sin 2 \alpha\left(\sin \frac{\delta}{2}+\sin \frac{3 \delta}{2}\right)\left(b+a^{*}\right)^{1 / 2}
\end{aligned}
$$




$$
\begin{array}{r}
\Sigma_{L}=\left[1-2\left(\frac{a}{b}\right)^{2} \sin ^{2} \alpha\right]\left(\cos ^{2} \delta+C_{x x} \sin ^{2} \delta\right) \\
+\left(\frac{a}{b}\right)^{2} \sin 2 \alpha \sin 2 \delta .
\end{array}
$$

The complete form of $\left(\sigma_{\theta \theta}^{L}\right)_{h}^{*}$ is shown in Appendix A. The stress intensity factor due to the incident field (15) can be easily obtained as follows

$$
\begin{gathered}
\left(K_{I}^{L}\right)_{i}(t, v, \delta)=\int_{0}^{v\left(t-t_{f}\right)} K_{I}^{F}\left(m=0, n=-1, t-t_{f^{-}}-\frac{x_{o}}{v}\right) \sigma_{\theta \theta}^{i} d x_{o} \\
=\frac{2 \sqrt{2} \sigma_{o} t_{f}^{1 / 2} \omega_{+}(d)\left(d^{*}-d\right)^{1 / 2}}{\sqrt{\pi}(1-a / d)^{1 / 2} d^{1 / 2}}\left[1-2\left(\frac{a}{b}\right)^{2} \sin ^{2}(\alpha-\delta)\right] .
\end{gathered}
$$

The first-order approximation of the mode I stress intensity factor, including the delay time effect for the kinking crack due to the normal loading on the original crack faces, is expressed in (22), and the solution for the step longitudinal stresswave loading is the sum of the contributions due to diffracted and incident fields given in (22) and (23),

$$
K_{I}^{L}=\left(K_{I}^{L}\right)_{d}+\left(K_{I}^{L}\right)_{i}
$$

Mode II Stress Intensity Factor. Following a similar analysis as in the mode I case, the mode II stress intensity factor due to the diffracted field (18) can be expressed as follows

$$
\begin{aligned}
& \left(K_{I I}^{L}\right)_{d}(t, v, \delta)=\int_{0}^{v\left(t-t_{f}\right) / t} K_{I I}^{F}(m= \\
& \left.-1, n=-t^{*}, t-t^{*}\right) \sigma_{\theta r}^{d}\left(\frac{1}{u}, \delta\right) d u \\
& =2 \sigma_{o}\left[\frac{2 t_{f}}{\pi d(1-b / d)}\right]^{1 / 2}\left\{u _ { + } ( d ) \left(d^{*}\right.\right. \\
& -d)^{1 / 2} \Lambda_{L}+\frac{d u_{+}(d) \Pi_{L}}{\left(c+a^{*}\right) S_{+}^{o}\left(a^{*}\right)}+ \\
& +\frac{d}{\pi\left(c+a^{*}\right) S_{+}^{o}\left(a^{*}\right)} \int_{d}^{d^{*}} \frac{\left(d^{*}-h\right)^{1 / 2}}{(h-d)^{1 / 2}}\left[\frac{u_{+}(d) \Pi_{L}}{(h-d)}\right. \\
& \left.\left.-\frac{u_{+}(h) d^{5 / 2}}{k(h-d)^{3}}\left(\sigma_{\theta r}^{L}\right)_{h}^{*}\right] d h\right\}+o(1),
\end{aligned}
$$

where

$$
\begin{aligned}
& \Pi_{L}=\frac{1}{4} {\left[1-2\left(\frac{a}{b}\right)^{2} \sin ^{2} \alpha\right]\left(\sin \frac{\delta}{2}+\sin \frac{3 \delta}{2}\right)\left(a+a^{*}\right)^{1 / 2} } \\
&-\frac{1}{4}\left(\frac{a}{b}\right)^{2} \sin 2 \alpha\left(\cos \frac{\delta}{2}+3 \cos \frac{3 \delta}{2}\right)\left(b+a^{*}\right)^{1 / 2} \\
& \Lambda_{L}=\frac{1}{2}\left[1-2\left(\frac{a}{b}\right)^{2} \sin ^{2} \alpha\right]\left(C_{x x}-1\right) \sin 2 \delta \\
&+\left(\frac{a}{b}\right)^{2} \sin 2 \alpha \cos 2 \delta .
\end{aligned}
$$

Details of $\left(\sigma_{\theta r}^{L}\right)_{h}^{*}$ are given in Appendix A. The mode II stress intensity factor due to the incident field (16) is

$$
\begin{gathered}
\left(K_{I I}^{L}\right)_{i}(t, v, \delta)=\int_{0}^{u\left(t-t_{f}\right)} K_{I I}^{F}\left(m=0, n=-1, t-t_{f}-\frac{x_{o}}{v}\right) \sigma_{\theta r}^{i} d x_{o} \\
=-\frac{2 \sqrt{2} \sigma_{o} t_{f}^{1 / 2} u_{+}(d)\left(d^{*}-d\right)^{1 / 2}}{\sqrt{\pi}(1-b / d)^{1 / 2} d^{1 / 2}}\left(\frac{a}{b}\right)^{2} \sin 2(\alpha-\delta) .
\end{gathered}
$$

Hence,

$$
K_{I I}^{L}=\left(K_{I I}^{L}\right)_{d}+\left(K_{I I}^{L}\right)_{i}
$$

\section{Crack Kinking Due to an Incident Transverse Wave}

Consider the incident transverse stress-wave of the form

$$
\sigma_{\bar{x} \bar{z}}^{i}=\sigma_{o} H(t+b r \sin (\alpha-\theta)) \text {. }
$$

The stress components in the polar coordinate for incident and diffracted fields of the stationary crack are

$$
\begin{gathered}
\sigma_{\theta \theta}^{i}=\sigma_{o} \sin 2(\alpha-\theta), \\
\sigma_{\theta r}^{i}=\sigma_{o} \cos 2(\alpha-\theta), \\
\sigma_{\theta \theta}^{d}=\frac{1}{2}(1-\cos 2 \theta) \sigma_{x x}^{T}+\frac{1}{2}(1+\cos 2 \theta) \sigma_{z z}^{T}-\sin 2|\theta| \sigma_{x z}^{T}, \\
\sigma_{\theta r}^{d}=\left[-\frac{1}{2} \sin 2|\theta| \sigma_{x x}^{T}+\frac{1}{2} \sin 2|\theta| \sigma_{z z}^{T}+\cos 2 \theta \sigma_{x z}^{T}\right] \operatorname{sgn}(\theta),
\end{gathered}
$$

where

$$
\sigma_{i j}^{T}=\sin 2 \alpha\left(\sigma_{i j}^{I}\right)^{*}+\cos 2 \alpha\left(\sigma_{i j}^{I I}\right)^{*} \operatorname{sgn}(\theta), i j=x x, z z, x z .
$$

Here $\left(\sigma_{i j}^{I}\right)^{*}$ and $\left(\sigma_{i j}^{I I}\right)^{*}$ are almost the same as $\sigma_{i j}^{I}$ and $\sigma_{i j}^{I I}$, which are defined in (4) and (7), respectively, except that $a^{*}$ should be replaced by $b^{*}=b \sin \alpha$.

The analysis for the stress intensity factor due to the incident transverse wave on a kinked crack proceeds in a similar manner as discussed in the previous section for the incident longitudinal wave. We will not report the solution procedure and only present the results.

\section{Mode I Stress Intensity Factor.}

$$
\begin{gathered}
K_{I}^{T}=\left(K_{I}^{T}\right)_{d}+\left(K_{I}^{T}\right)_{i}, \\
\left(K_{I}^{T}\right)_{d}(t, v, \delta)=2 \sigma_{o}\left[\frac{2 t_{f}}{\pi d(1-a / d)}\right]^{1 / 2}\left\{-\omega_{+}(d)\left(d^{*}-d\right)^{1 / 2} \Sigma_{T}\right. \\
+\frac{d \omega_{+}(d) \Gamma_{T}}{\left(c+b^{*}\right) S_{+}^{o}\left(b^{*}\right)}+\frac{d}{\pi\left(c+b^{*}\right) S_{+}^{o}\left(b^{*}\right)} \int_{d}^{d^{*}} \frac{\left(d^{*}-h\right)^{1 / 2}}{(h-d)^{1 / 2}} \\
\left.\left[\frac{\omega_{+}(d) \Gamma_{T}}{(h-d)}-\frac{\omega_{+}(h) d^{5 / 2}}{k(h-d)^{3}}\left(\sigma_{\theta \theta}^{T}\right)_{h}^{*}\right] d h\right\}+o(1)
\end{gathered}
$$

where

$\Gamma_{T}=\sin 2 \alpha \cos ^{3} \frac{\delta}{2}\left(a+b^{*}\right)^{1 / 2}$

$$
\begin{gathered}
-\frac{3}{4} \cos 2 \alpha\left(\sin \frac{\delta}{2}+\sin \frac{3 \delta}{2}\right)\left(b+b^{*}\right)^{1 / 2}, \\
\Sigma_{T}=\sin 2 \alpha\left(\cos ^{2} \delta+C_{x x} \sin ^{2} \delta\right)-\cos 2 \alpha \sin 2 \delta,
\end{gathered}
$$

and

$\left(K_{I}^{T}\right)_{i}(t, v, \delta)=\frac{2 \sqrt{2} \sigma_{o} t_{f}^{1 / 2} \omega_{+}(d)\left(d^{*}-d\right)^{1 / 2}}{\sqrt{\pi}(1-a / d)^{1 / 2} d^{1 / 2}} \sin 2(\alpha-\delta)$

Mode II Stress Intensity Factor.

$$
\begin{gathered}
K_{I I}^{T}=\left(K_{I I}^{T}\right)_{d}+\left(K_{I I}^{T}\right)_{i}, \\
\left(K_{I I}^{T}\right)_{d}(t, v, \delta)=2 \sigma_{o}\left[\frac{2 t_{f}}{\pi d(1-b / d)}\right]^{1 / 2}\left\{u_{+}(d)\left(d^{*}-d\right)^{1 / 2} \Lambda_{T}\right. \\
+\frac{d u_{+}(d) \Pi_{T}}{\left(c+b^{*}\right) S_{+}^{o}\left(b^{*}\right)}+ \\
+\frac{d}{\pi\left(c+b^{*}\right) S_{+}^{o}\left(b^{*}\right)} \int_{d}^{d^{*}} \frac{\left(d^{*}-h\right)^{1 / 2}}{(h-d)^{1 / 2}}\left[\frac{u_{+}(d) \Pi_{T}}{(h-d)}\right. \\
\left.\left.-\frac{u_{+}(h) d^{5 / 2}}{k(h-d)^{3}}\left(\sigma_{\theta r}^{T}\right)_{h}^{*}\right] d h\right\}+o(1),
\end{gathered}
$$


where

$$
\begin{aligned}
\Pi_{T}=\frac{1}{4} \sin 2 \alpha\left(\sin \frac{\delta}{2}+\sin \frac{3 \delta}{2}\right)\left(a+b^{*}\right)^{1 / 2} \\
+\frac{1}{4} \cos 2 \alpha\left(\cos \frac{\delta}{2}+3 \cos \frac{3 \delta}{2}\right)\left(b+b^{*}\right)^{1 / 2} \\
\Lambda_{T}=\frac{1}{2} \sin 2 \alpha\left(C_{x x}-1\right) \sin 2 \delta-\cos 2 \alpha \cos 2 \delta
\end{aligned}
$$

and

$$
\left(K_{I I}^{T}\right)_{i}(t, v, \delta)=\frac{2 \sqrt{2} \sigma_{o} t_{f}^{1 / 2} \dot{u}_{+}(d)\left(d^{*}-d\right)^{1 / 2}}{\sqrt{\pi}(1-b / d)^{1 / 2} d^{1 / 2}} \cos 2(\alpha-\delta)
$$

Details of $\left(\sigma_{\theta \theta}^{T}\right)_{h}^{*}$ and $\left(\sigma_{\theta r}^{T}\right)_{h}^{*}$ are given in Appendix B.

\section{Special Cases and Numerical Results}

Some special cases are discussed to give simple closed-form results and can be used as a check for the numerical calculation of the general cases. For the kinking angle $\delta=0$, the new crack propagates straight out of the original crack. The stress intensity factor can then be simplified as

$$
\begin{gathered}
K_{I}^{L}=2 \sqrt{\frac{2}{\pi}} \sigma_{o}\left(1-2 \frac{a^{* 2}}{b^{2}}\right) \omega_{+}^{o}\left(a^{*}\right) \kappa_{I}(d)\left[t+v\left(t-t_{f}\right) a^{*}\right]^{1 / 2} \\
K_{I I}^{L}=-2 \sqrt{\frac{2}{\pi} \sigma_{o} \frac{a^{2}}{b^{2}} \sin 2 \alpha u_{+}^{o}\left(a^{*}\right) \kappa_{I I}(d)\left[t+v\left(t-t_{f}\right) a^{*}\right]^{1 / 2}} \\
K_{I}^{T}=2 \sqrt{\frac{2}{\pi} \sigma_{o} \sin 2 \alpha \omega_{+}^{o}\left(b^{*}\right) \kappa_{I}(d)\left[t+v\left(t-t_{f}\right) b^{*}\right]^{1 / 2}} \\
K_{I I}^{T}=2 \sqrt{\frac{2}{\pi}} \sigma_{o} \cos 2 \alpha u_{+}^{o}\left(b^{*}\right) \kappa_{I I}(d)\left[t+v\left(t-t_{f}\right) b^{*}\right]^{1 / 2}
\end{gathered}
$$

where

$$
\begin{aligned}
\kappa_{I}(d) & =\frac{d}{S_{+}(d)\left(d+c_{2}\right)(1-a / d)^{1 / 2}} \\
\kappa_{I I}(d) & =\frac{d}{S_{+}(d)\left(d+c_{2}\right)(1-b / d)^{1 / 2}}
\end{aligned}
$$

The functions $\kappa_{I}(d)$ and $\kappa_{I I}(d)$ depend only on the crack speed $v=1 / d$ and material properties. The values of $\kappa_{I}(d)$ and $k_{I I}(d)$ decrease from unity at $v=0$ to zero when the crack speed reaches the Rayleigh wave speed. It is worth noting that the solution in (39)-(42) provides exact results, without any approximation made in this case and reduce to the same results obtained by Freund (1974) for a crack propagating straight.

If one wants to study the criterion for a crack kinking event, it is clear that the most significant time scale involved should be in the region when crack kinking has just occurred, in other words, $t-t_{f}<<1$. The field quantities change very rapidly at this time period and it certainly plays an important role in the crack kinking events. The stress intensity factor just after the kinking occurs has the form

$$
K_{I}^{L}=2 \sigma_{o}\left[\frac{2 t d}{\pi(1-a / d)}\right]^{1 / 2} \frac{\omega_{+}(d) \Gamma_{L}}{\left(c+a^{*}\right) S_{+}^{o}\left(a^{*}\right)}
$$

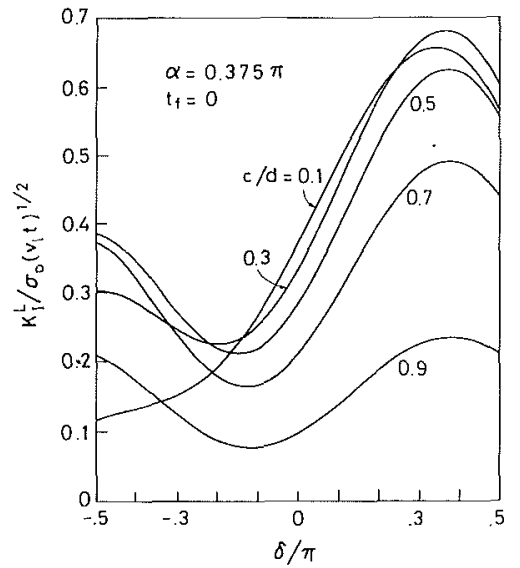

Fig. 2 The normalized mode / stress intensity factor versus kinking angle due to incident longitudinal stress-wave loading for $t_{f}=0$ and $\alpha$ $=0.375 \pi$

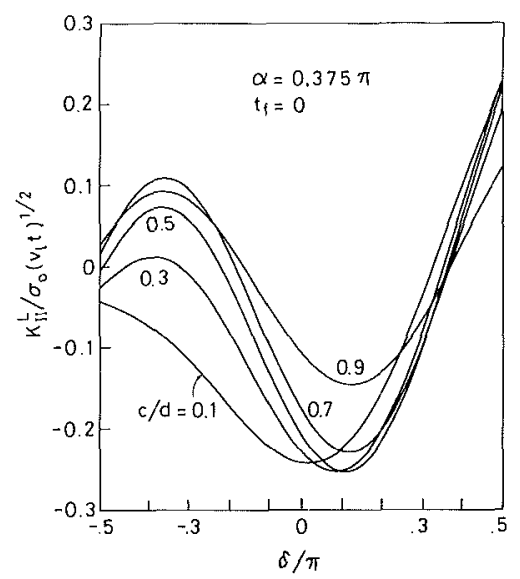

Fig. 3 The normalized mode II stress intensity factor versus kinking angle due to incident longitudinal stress-wave loading for $t_{t}=0$ and $a$ $=0.375 \pi$

$$
\begin{aligned}
& K_{I I}^{L}=2 \sigma_{o}\left[\frac{2 t d}{\pi(1-b / d)}\right]^{1 / 2} \frac{u_{+}(d) \Pi_{L}}{\left(c+a^{*}\right) S_{+}^{o}\left(a^{*}\right)}, \\
& K_{I}^{T}=2 \sigma_{o}\left[\frac{2 t d}{\pi(1-a / d)}\right]^{1 / 2} \frac{\omega_{+}(d) \Gamma_{T}}{\left(c+b^{*}\right) S_{+}^{o}\left(b^{*}\right)}, \\
& K_{I I}^{T}=2 \sigma_{o}\left[\frac{2 t d}{\pi(1-b / d)}\right]^{1 / 2} \frac{u_{+}(d) \Pi_{T}}{\left(c+b^{*}\right) S_{+}^{o}\left(b^{*}\right)} .
\end{aligned}
$$

Just before kinking, the crack is stationary and the stress intensity factor can be obtained by taking the limit $t \rightarrow t_{f}$ and $d \rightarrow \infty$ in equations (39)-(42) or by taking the limit $\delta \rightarrow 0$ and $d \rightarrow \infty$ in equations (43)-(46).

$$
\begin{gathered}
\left(K_{I}^{L}\right)_{s}=2 \sigma_{o}\left[1-2(a / b)^{2} \sin ^{2} \alpha\right](2 t / \pi)^{1 / 2} \omega_{+}^{o}\left(a^{*}\right), \\
\left(K_{I I}^{L}\right)_{s}=-2 \sigma_{o}(a / b)^{2} \sin 2 \alpha(2 t / \pi)^{1 / 2} u_{+}^{o}\left(a^{*}\right), \\
\left(K_{I}^{T}\right)_{s}=2 \sigma_{o} \sin 2 \alpha(2 t / \pi)^{1 / 2} \omega_{+}^{o}\left(b^{*}\right), \\
\left(K_{I I}^{T}\right)_{s}=2 \sigma_{o} \cos 2 \alpha(2 t / \pi)^{1 / 2} u_{+}^{o}\left(b^{*}\right) .
\end{gathered}
$$

Because the crack-tip speed changes discontinuously at $t=$ $t_{f}$, the stress intensity factor just before and after the kinked crack has a jump, whose magnitude depends on the crack speed $v$, the incident angle $\alpha$, and the kinked angle $\delta$.

If we examine the special case of no finite delay time, that is the crack kinks at the instant the incident stress wave strikes the original crack-tip, the solution of (24), (27), (33), and (36) will reduce to the result of Achenbach, Kuo, and Dempsey (1984). Figures 2 and 3 show the dimensionless mode I and 


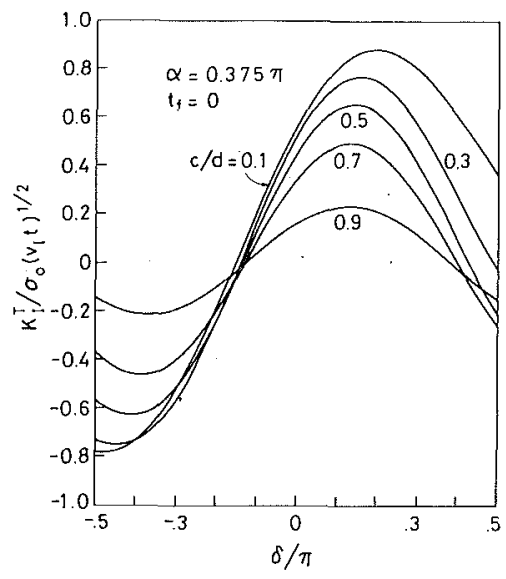

Fig. 4 The normalized mode I stress intensity factor versus kinking angle due to incident tránsverse stress-wave loading for $t_{t}=0$ and $\alpha$ $=0.375 \pi$

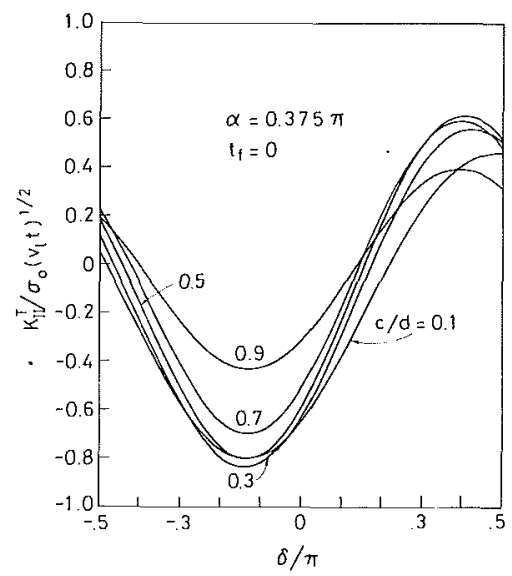

Fig. 5 The normalized mode II stress intensity factor versus kinking angle due to incident transverse stress-wave loading for $t_{t}=0$ and $\alpha$ $=0.375 \pi$

\begin{tabular}{|c|c|c|c|c|c|c|c|c|}
\hline & $\mathrm{v} / \mathrm{v}_{\mathrm{B}} \delta$ & & 0 & .0625 & .125 & .25 & .375 & .485 \\
\hline & & (1) & 1.0070 & .9870 & .9291 & .7241 & .4625 & .2482 \\
\hline & 0.1 & (2) & .9991 & .9797 & .9234 & .7219 & .4610 & .2829 \\
\hline & & (3) & .9997 & .9808 & .9247 & .7239 & .4622 & .2448 \\
\hline & & (1) & .8484 & .8295 & .7747 & .5818 & .3387 & .1435 \\
\hline & 0.3 & (2) & .8625 & .8435 & .7884 & .5937 & .3481 & .1932 \\
\hline$K_{I}^{L}$ & & (3) & .8623 & .8434 & .7884 & .5945 & .3495 & .1537 \\
\hline$\sigma_{0}\left(v_{l} t\right)^{1 / 2}$ & & (1) & .7078 & .6911 & .6427 & .4744 & .2674 & .1068 \\
\hline & 0.5 & (2) & .7023 & .6856 & .6373 & .4695 & .2637 & .1356 \\
\hline & & (3) & .7019 & .6853 & .6372 & .4701 & .2650 & .1069 \\
\hline & & (1) & .5139 & .5011 & .4644 & .3385 & .1895 & .0805 \\
\hline & 0.7 & (2) & .5043 & .4916 & .4549 & .3294 & .1810 & .0899 \\
\hline & & (3) & .5040 & .4913 & .4548 & .3299 & .1821 & .0738 \\
\hline \multirow[b]{6}{*}{$K_{{ }_{I}}^{L}$} & & (1) & 0 & .1287 & .2465 & .4131 & .4564 & .3952 \\
\hline & 0.1 & (2) & 0 & .1257 & .2410 & .4047 & .4458 & .3848 \\
\hline & & (3) & 0 & .1251 & .2397 & .4030 & .4439 & .3787 \\
\hline & & (1) & 0 & .1342 & .2564 & .4260 & .4636 & .3962 \\
\hline & 0.3 & (2) & 0 & .1276 & .2436 & .4023 & .4299 & .3565 \\
\hline & & (3) & 0 & .1271 & .2427 & .4009 & .4285 & .3514 \\
\hline \multirow[t]{6}{*}{$\sigma_{0}(v, t)^{t / 2}$} & & (1) & 0 & .1245 & .2373 & .3909 & .4202 & .3551 \\
\hline & 0.5 & (2) & 0 & .1197 & .2277 & .3711 & .3887 & .3155 \\
\hline & & (3) & 0 & .1192 & .2268 & .3697 & .3874 & .3115 \\
\hline & & (1) & $\theta$ & $\therefore 1069$ & .2031 & .3301 & .3479 & .2890 \\
\hline & 0.7 & (2) & 0 & .1029 & .1951 & .3136 & .3220 & .2570 \\
\hline & & (3) & 0 & .1025 & .1943 & .3123 & .3209 & .2543 \\
\hline
\end{tabular}

mode II stress intensity factor which is valid for $t_{f}=0$ and incident longitudinal stress wave angle $\alpha=3 \pi / 8$. Note that for all calculations in this paper, a Poisson's ratio of 0.25 is used which gives a ratio of wave speed $v_{l}=\sqrt{3} v_{s}, v_{l}=1.884 v_{R}$. The numerical results of the mode $I$ and mode II stress intensity factor due to incident transverse stress wave for $t_{f}=0$ are 


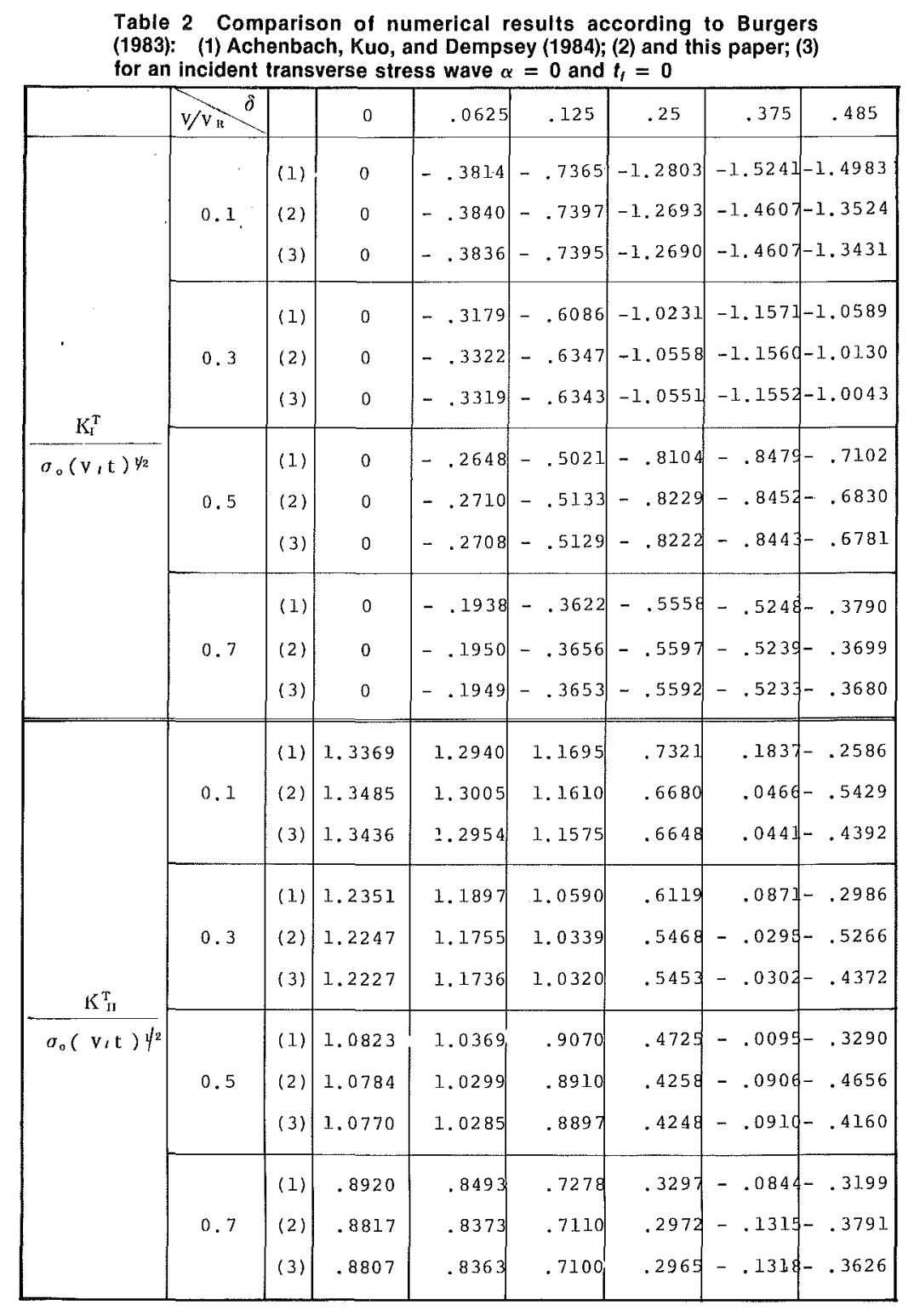

shown in Figs. 4 and 5. These results agree very well with the numerical results presented by Achenbach, Kuo, and Dempsey (1984). A comparison with the numerical results with Achenbach, Kuo, and Dempsey (1984) and Burgers (1983) is shown in Tables 1 and 2, for both incident longitudinal and transverse waves with $\alpha=0$. It shows that the first-order approximation method used in this paper agrees quite well with the numerical results of Burgers (1983). For the important range of kinking angles $0<\delta<\pi / 4$, the error introduced by using this approximate method is within 3 percent for mode I stress intensity factor and 5 percent for mode II stress intensity factor due to incident longitudinal wave. For incident transverse wave, the error is within 3 percent for mode I and 10 percent for mode II. This good agreement suggests that the wedge geometry of the kinked crack has only a minor effect on the calculation of the dynamic stress intensity factor.

In order to investigate the stress intensity factor for the whole propagation event of the kinked crack, the normalized time $t_{f} / t$ is chosen as the parameter. The instant of initiation of the kink is at $t_{f} / t=1$, while $t_{f} / t=0$ corresponds to the time when the kinked crack has propagated for an infinite time compared to the delay time. The nondimensional stress inten- sity factor for mode I and mode II versus $t_{f} / t$ for incident longitudinal wave (or transverse wave) for incident angle $\alpha=$ $0.375 \pi$, and kinking angles $\delta= \pm 0.125 \pi$ are calculated and plotted in Figs. 6-9. As we can see from these figures, the dimensionless stress intensity factor is significantly different for $t_{f} / t=0$ and $t_{f} / t=1$ for some cases. The mode II stress intensity factor has a stronger dependence on delay time effect than mode I case.

\section{Energy Fluxes and Kinking Criterion}

For mixed mode I-II fracture, the energy flux into the propagating crack-tip can be written in terms of the corresponding dynamic stress intensity factors in the form

$$
\begin{aligned}
E & =-\frac{b^{2}}{2 \mu d^{3} R(d)}\left[\left(1-\frac{a^{2}}{d^{2}}\right)^{1 / 2}\left(K_{I}\right)^{2}+\left(1-\frac{b^{2}}{d^{2}}\right)^{1 / 2}\left(K_{I}\right)^{2}\right] \\
& =\frac{\sigma_{o}^{2} t}{2 \mu b^{2}} E^{*}
\end{aligned}
$$

where 


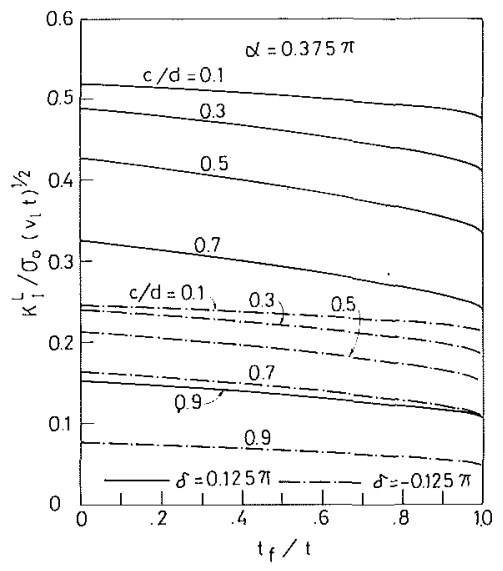

Fig. 6 The time history of mode I stress intensity factor for $\delta=\pi / 8$, $-\pi / 8$ due to incident longitudinal stress-wave loading for $\alpha=0.375 \pi$

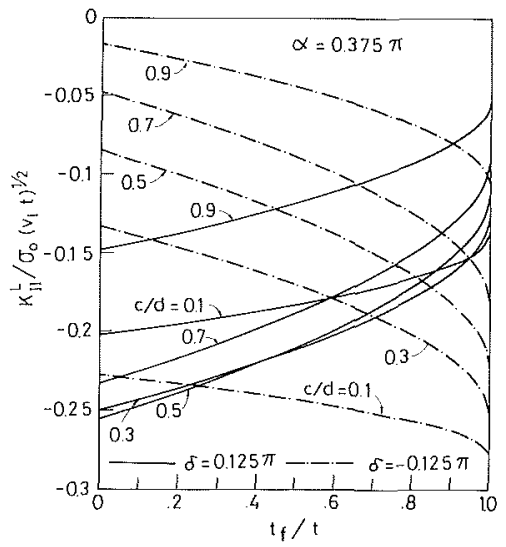

Fig. 7 The time history of mode II stress intensity factor for $\delta=\pi / 8$, $-\pi / 8$ due to incident longitudinal stress-wave loading for $\alpha=0.375 \pi$

$$
R(d)=\left(\frac{b^{2}}{d^{2}}-2\right)^{2}-4\left(1-\frac{a^{2}}{d^{2}}\right)^{1 / 2}\left(1-\frac{b^{2}}{d^{2}}\right)^{1 / 2}
$$

In the following calculations of $E^{*}$, the stress intensity factor might show a negative value. A negative mode I stress intensity factor would correspond to the contact of the crack faces near the crack-tip. Hence, we would set $K_{I}$ identically equal to zero whenever the calculations show it to be negative. The effect on the negative mode II stress intensity factor may be ignored under the smooth, frictionless crack faces assumption. If the maximum energy release rate criterion is accepted as the kinking condition, then the combination of the kinking angle and the crack speed can be determined at which the energy flux into the propagating crack-tip achieves a maximum value. For an incident longitudinal wave, Fig. 10 shows the kinking angle and the crack-tip speed at which $E^{*}$ attains its maximum value for various values of $\alpha$ for $t_{f}=0$. The predicted kinked crack speed increases as the incident angle $\alpha$ increase. For the general case, the delay time is not zero, it is shown in Fig. 11 for incident angle $\alpha=\pi / 4$ that the kinking angle and crack tip speed for $E^{*}$ to achieve its maximum value $E_{\max }^{*}$ of the whole time history.

\section{Conclusion}

With the inclusion of a delay time, the solution of the fracture problem becomes a great deal more realistic physically and more closely models real material response. An approximate method that ignores the corner geometry of the kink angle is used to construct the mixed-mode stress intensity factor for incident longitudinal and transverse stress-wave loading.

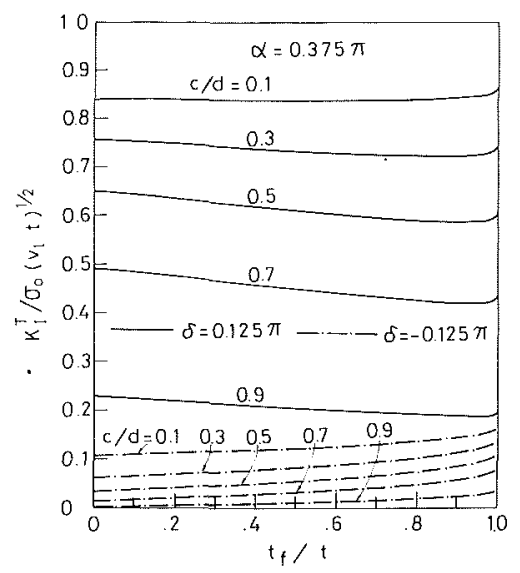

Fig. 8 The time history of mode I stress intensity factor for $\delta=\pi / 8$, $-\pi / 8$ due to incident transverse stress-wave loading for $\alpha=0.375 \pi$

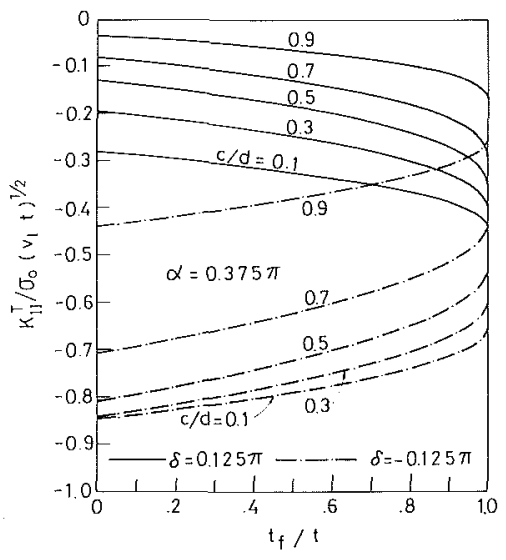

Fig. 9 The time history of mode II stress intensity factor for $\delta=\pi / 8$, $-\pi / 8$ due to incident transverse stress-wave loading for $\alpha=0.375 \pi$

A very satisfactory result is obtained when compared with the numerical results with no delay time effect. This good agreement suggests that the wedge geometry of the kinked crack has only a minor effect on the calculation of the dynamic stress intensity factor. Hence, for important range of kinking angles, the elastodynamic crack kinking stress intensity factors are affected more by the loading of the new crack faces than by the wedge geometry.

The influence of the delay time effect on the calculation of the dynamic stress intensity factor for kinking crack can be obtained from Figs. 6-9. The figures show that the mode I stress intensity factor is weakly dependent on delay time, while the mode II stress intensity factor has a stronger dependence on delay time. For the kinking angle $\delta=0$, the crack propagates straight out of the original crack, the stress intensity factors are expressed in (39)-(42). An interesting result is that $K_{I}^{L}$ and $K_{I I}^{T}$ are independent of the delay time for $\alpha=0$.

Frequently, an energy-based fracture criterion is used to look at the initiation of crack-tip motion. The criterion is based on the assumption that the energy release rate at initiation of fracture is a material parameter. For static fracture under small-scale yielding conditions this is well established, but for dynamic fracture, it is not clear that this is a suitable criterion beyond the initiation phase. It is unfortunately not yet clear from experimental results what is a suitable criterion for a bifurcation event. Recent attempts have been made to determine the condition for crack branching from theoretical analysis of the elastodynamic field quantities near the tips of the branches. With these theoretical results for the stress intensity factor of the kinking crack, an attempt can be made to de- 


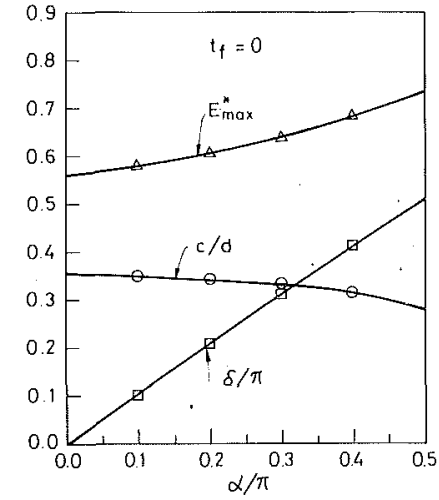

Fig. 10 Kinking angle and crack-tip speed for $E_{\max }^{*}$ of incident various angles of longitudinal wave

termine the kink angle and the new kinked crack speed using different fracture criteria and to compare them with the experimental results available. For this paper, the maximum energy release rate is adopted for the kinking criterion. This energy criterion suggests that the crack will choose to propagate in the direction and at velocity for which the energy flux into the crack tip has a maximum value. For the special case $t_{f}=$ 0 , the general features are that the kinked crack speed $v$ increases as the incident stress wave angle $\alpha$ increases for $E^{*}$ to achieve its maximum value $E_{\max }^{*}$, and the kinked angle $\delta$ is just slightly larger than $\alpha$. For the general case, the kinking angle $\delta$ is approximately constant until $t_{f} / t$ reaches 0.8 . In general, $v$ and $\delta$ are slightly smaller than the corresponding values obtained by assuming no delay time. For incident stress-wave parallel to the crack faces $(\alpha=0)$, the energy criterion predicts that the crack will tend to propagate straight ahead of the original crack which has been observed in experiments, see Ravi-Chandar and Knauss (1984a, b, c, d).

The complete solutions available for the kinked crack geometry that include the corner effect are still restricted to no delay time. There are no other results that can be used to judge the accuracy of the approximation in this paper when the delay time effect is included in the whole kinking angle range. It has been shown, that for $\delta=0$, the results are exact. It is believed that this approximate method still gives a quite good accuracy for small kinked angles.

\section{Acknowledgments}

The research support of the R.O.C. National Science Council through Grant NSC76-0401-E002-17 at National Taiwan University is gratefully acknowledged.

\section{References}

Achenbach, J. D., 1970, "Crack Propagation Generated by a Horizontally Polarized Shear Wave," J. Mech. Phys. Solids, Vol. 18, pp. 245-259.

Achenbach, J. D., and Kuo, M. K., 1985, "Conditions for Crack Kinking under Stress-Wave Loading," Engineering Fracture Mechanics, Vol. 22, pp. 165180.

Achenbach, J. D., Kuo, M. K., and Dempsey, J. P., 1984, "Mode III and Mixed Mode I-II Crack Kinking under Stress-Wave Loading," Int. J. Solids Structures, Vol. 20, pp. 395-410.

Achenbach, J. D., and Varatharajulu, 1974, "Skew Crack Propagation at the Diffraction of a Transient Stress Wave," Quarterly Appl. Math., Vol. 32, pp. 123-135.

Burgers, P., 1982, "Dynamic Propagation of a Kinked or Bifurcated Crack in Anti-plane Strain," ASME Journal, of Applied Mechanics, Vol. 49, pp. 371-376.

Burgers, P., 1983, "Dynamic Kinking of a Crack in Plane Strain," Int. J. Solids Structures, Vol. 19, pp. 735-752.

Burgers, P., and Dempsey, J. P., 1982, "Two Analytical Solutions for Dynamic Crack Bifurcation in Anti-plane Strain," ASME Journal of ApPLIED Mechanics, Vol. 49 , pp. $366-370$.

Burgers, P., and Dempsey, J. P., 1984, "Plane Strain Dynamic Crack Bifurcation," Int. J. Solids Structures, Vol. 20, pp. 609-618.

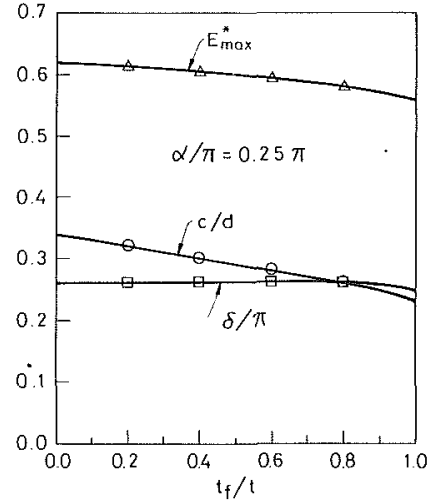

Fig. 11 Kinking angle and crack-tip speed for $E_{\max }^{*}$ in whole time history for $\alpha=\pi / 4$

Dempsey, J. P., Kuo, M. K., and Achenbach, J. D., 1982, "Mode III Crack Kinking under Stress Wave Loading," Wave Motion, Vol, 4, pp. 181-190.

Dempsey, J. P., Kuo, M. K., and Bentley, D. L.,1986, "Dynamic Effects in Mode III Crack Bifurcation," Int. J. Solids Structures, Vol. 22, pp. 333-353.

Freund, L. B., 1973, "Crack Propagation in an Elastic Solid Subjected to General Loading-III, Stress Wave Loading," J. Mech. Phys. Solids, Vol. 21, pp. 47-61.

Freund, L. B., 1974, "Crack Propagation in an Elastic Solid Subjected to General Loading-IV, Obliquely Incident Stress Pulse," J. Mech. Phys. Solids, Vol. 22, pp. 137-146.

Ma, C. C., and Burgers, P., 1986, "Mode III Crack Kinking with Delay Time: An Analytical Approximation," Int. J. Solids Structures, Vol. 22, pp. 883-899.

Ma, C. C., and Burgers, P., 1987, "Dynamic Mode I and Mode II Crack Kinking Including Delay Time Effects," Int. J. Solids Structures, Vol. 23, pp. 897-918.

Ravi-Chandar, K., and Knauss, W. G., 1984a, "An Experimental Investigation into Dynamic Fracture: I. Crack Initiation and Arrest," International Journal of Fracture, Vol. 25, pp. 247-262.

Ravi-Chandar, K., and Knauss, W. G., 1984b, "An Experimental Investigation into Dynamic Fracture: II. Microstructural Aspects," International Journal of Fracture, Vol. 26, pp. 65-80.

Ravi-Chandar, K., and Knauss, W. G., 1984c, "An Experimental Investigation into Dynamic Fracture: III. On Steady-State Crack Propagation and Crack Branching,"' International Journal of Fracture, Vol. 26, pp. 141-154.

Ravi-Chandar, K., and Knauss, W. G., 1984d, "An Experimental Investigation into Dynamic Fracture: IV. On the Interaction of Stress Waves with Propagating Cracks," International Journal of Fracture, Vol. 26, pp. 189-200.

\section{A P P E N D I X A}

$\left(\sigma_{\theta \theta}^{L}\right)_{h}^{*}=\left(a+a^{*}\right)^{1 / 2}\left[1-2\left(\frac{a}{b}\right)^{2} \sin ^{2} \alpha\right]\left(\sigma_{\theta \theta}^{I}\right)_{h}^{*}$

$$
-\left(b+a^{*}\right)^{1 / 2}\left(\frac{a}{b}\right)^{2} \sin 2 \alpha\left(\sigma_{\theta \theta}^{I I}\right)_{h}^{*} \operatorname{sgn}(\delta) \text {. }
$$

$$
\begin{aligned}
\left(\sigma_{r \theta}^{L}\right)_{h}^{*}=\left(a+a^{*}\right)^{1 / 2}\left[1-2\left(\frac{a}{b}\right)^{2} \sin ^{2} \alpha\right]\left(\sigma_{r \theta}^{I}\right)_{h}^{*} \\
-\left(b+a^{*}\right)^{1 / 2}\left(\frac{a}{b}\right)^{2} \sin 2 \alpha\left(\sigma_{r \theta}^{I I}\right)_{h}^{*} \operatorname{sgn}(\delta) .
\end{aligned}
$$

$$
\begin{gathered}
\left(\sigma_{\theta \theta}^{p}\right)_{h}^{*}=\frac{1}{2}(1-\cos 2 \delta)\left(\sigma_{x x}^{p}\right)_{h}^{*}+\frac{1}{2}(1 \\
\quad+\cos 2 \delta)\left(\sigma_{z z}^{p}\right)_{h}^{*}-\sin |2 \delta|\left(\sigma_{x z}^{p}\right)_{h}^{*}, p=I \text { or } I I \\
\left(\sigma_{\theta r}^{q}\right)_{h}^{*}=\left[-\frac{1}{2} \sin |2 \delta|\left(\sigma_{x x}^{q}\right)_{h}^{*}+\frac{1}{2} \sin |2 \delta|\left(\sigma_{z z}^{q}\right)_{h}^{*}\right. \\
\left.+\cos 2 \delta\left(\sigma_{x z}^{q}\right)_{h}^{*}\right] \operatorname{sgn}(\delta), q=I \text { or } I I
\end{gathered}
$$

and 


$$
\begin{aligned}
& \left(\sigma_{z z}^{I}\right)_{h}^{*}=\operatorname{Im}\left\{-\frac{\left[2 \Theta_{a}^{2}-b^{2}(v h-1)^{2}\right]^{2} \Omega_{a}}{\Psi_{a}\left[a(v h-1)+\theta_{a}\right]^{1 / 2}}\right. \\
& \left.-\frac{4 \Theta_{b}^{2}\left[a(v h-1)-\Theta_{b}\right]^{1 / 2}\left[b^{2}(v h-1)^{2}-\theta_{b}^{2}\right]^{1 / 2} \Omega_{b}}{\Psi_{b}}\right\}, \\
& \left(\sigma_{x z}^{I}\right)_{h}^{*}=\operatorname{Im}\left\{-\frac{2 \Theta_{a}\left[a(v h-1)-\Theta_{a}\right]^{1 / 2}\left[2 \theta_{a}^{2}-b^{2}(v h-1)^{2}\right] \Omega_{a}}{\Psi_{a}}+\right. \\
& \left.+\frac{2 \Theta_{b}\left[a(v h-1)-\theta_{b}\right]^{1 / 2}\left[2 \theta_{b}^{2}-b^{2}(v h-1)^{2}\right] \Omega_{b}}{\Psi_{b}}\right\}, \\
& \left(\sigma_{x x}^{I}\right)_{h}^{*}=\operatorname{Im}\left\{\frac{\left.2 \Theta_{a}^{2}-b^{2}(v h-1)^{2}\right]\left[2 \Theta_{a}^{2}+\left(b^{2}-2 a^{2}\right)(v h-1)^{2}\right] \Omega_{a}}{\Psi_{a}\left[a(v h-1)+\theta_{a}\right]^{1 / 2}}+\right. \\
& \left.+\frac{4 \Theta_{b}^{2}\left[a(v h-1)-\Theta_{b}\right]^{1 / 2}\left[b^{2}(v h-1)^{2}-\Theta_{b}^{2}\right]^{1 / 2} \Omega_{b}}{\Psi_{b}}\right\}, \\
& \left(\sigma_{z z}^{I I}\right)_{h}^{*}=\operatorname{Im}\left\{\frac{2 \Theta_{a}\left[b(v h-1)-\Theta_{a}\right]^{1 / 2}\left[b^{2}(v h-1)^{2}-2 \Theta_{a}^{2}\right] \Omega_{a}}{\Psi_{a}}-\right. \\
& \left.-\frac{2 \Theta_{b}\left[b(v h-1)-\theta_{b}\right]^{1 / 2}\left[b^{2}(v h-1)^{2}-2 \theta_{b}^{2}\right] \Omega_{b}}{\Psi_{b}}\right\}, \\
& \left(\sigma_{x z}^{I I}\right)_{h}^{*}=\operatorname{Im}\left\{-\frac{4 \Theta_{a}^{2}\left[b(v h-1)-\Theta_{a}\right]^{1 / 2}\left[a^{2}(v h-1)^{2}-\theta_{a}^{2}\right]^{1 / 2} \Omega_{a}}{\Psi_{a}}\right. \\
& \left.-\frac{\left[2 \theta_{b}^{2}-b^{2}(v h-1)^{2}\right]^{2} \Omega_{b}}{\Psi_{b}\left[b(v h-1)+\theta_{b}\right]^{1 / 2}}\right\} \\
& \left(\sigma_{x x}^{I I}\right)_{h}^{*}=\operatorname{Im}\left\{\frac{2 \Theta_{a}\left[b(v h-1)-\theta_{a}\right]^{1 / 2}\left[2 \Theta_{a}^{2}+\left(b^{2}-2 a^{2}\right)(v h-1)^{2}\right] \Omega_{a}}{\Psi_{a}}\right. \\
& \left.+\frac{2 \Theta_{b}\left[b(v h-1)-\theta_{b}\right]^{1 / 2}\left[b^{2}(v h-1)^{2}-2 \Theta_{b}^{2}\right] \Omega_{b}}{\Psi_{b}}\right\}
\end{aligned}
$$

where

$$
\begin{gathered}
\Omega_{a}=-\left[h^{2}-a^{2}(v h-1)^{2}\right]^{1 / 2} \cos \delta+i h \sin |\delta|, \\
\Omega_{b}=-\left[h^{2}-b^{2}(v h-1)^{2}\right]^{1 / 2} \cos \delta+i h \sin |\delta|, \\
\Theta_{a}=-h \cos \delta+i\left[h^{2}-a^{2}(v h-1)^{2}\right]^{1 / 2} \sin |\delta|, \\
\Theta_{b}=-h \cos \delta+i\left[h^{2}-b^{2}(v h-1)^{2}\right]^{1 / 2} \sin |\delta|, \\
\Psi_{a}=\left[h^{2}-a^{2}(v h-1)^{2}\right]^{1 / 2}\left[\Theta_{a}-a(v h-1) \sin \alpha\right] \\
{\left[\Theta_{a}-c(v h-1)\right] S_{-}^{o}\left(\Theta_{a} /(v h-1)\right),} \\
\Psi_{b}=\left[h^{2}-b^{2}(v h-1)^{2}\right]^{1 / 2}\left[\Theta_{b}-a(v h-1) \sin \alpha\right] \\
{\left[\theta_{b}-c(v h-1)\right] S_{-}^{o}\left(\theta_{b} /(v h-1)\right) .}
\end{gathered}
$$

\section{A P P E N D I X B}

$\left(\sigma_{\theta \theta}^{T}\right)_{h}^{*}=\left(a+b^{*}\right)^{1 / 2} \sin 2 \alpha\left(\sigma_{\theta \theta}^{I}\right)_{h}^{*}+\left(b+b^{*}\right)^{1 / 2} \cos 2 \alpha\left(\sigma_{\theta \theta}^{I I}\right)_{h}^{*} \operatorname{sgn}(\delta)$.

$\left(\sigma_{r \theta}^{T}\right)_{h}^{*}=\left(a+b^{*}\right)^{1 / 2} \sin 2 \alpha\left(\sigma_{r \theta}^{I}\right)_{h}^{*}+\left(b+b^{*}\right)^{1 / 2} \cos 2 \alpha\left(\sigma_{r \theta}^{I I}\right)_{h}^{*} \operatorname{sgn}(\delta)$.

$\left(\sigma_{\theta \theta}^{p}\right)_{h}^{*}=\frac{1}{2}(1-\cos 2 \delta)\left(\sigma_{x x}^{p}\right)_{h}^{*}+\frac{1}{2}(1+\cos 2 \delta)\left(\sigma_{z z}^{p}\right)_{h}^{*}$

$$
-\sin |2 \delta|\left(\sigma_{x z}^{p}\right)_{h}^{*}, p=I \text { or } I I
$$

$\left(\sigma_{\theta r}^{q}\right)_{h}^{*}=\left[-\frac{1}{2} \sin |2 \delta|\left(\sigma_{x x}^{q}\right)_{h}^{*}+\frac{1}{2} \sin |2 \delta|\left(\sigma_{z z}^{q}\right)_{h}^{*}\right.$

$$
\left.+\cos 2 \delta\left(\sigma_{x z}^{q}\right)_{h}^{*}\right] \operatorname{sgn}(\delta), q=I \text { or } I I,
$$

where $\left(\sigma_{\theta \theta}^{I}\right)_{h}^{*},\left(\sigma_{r \theta}^{I}\right)_{h}^{*},\left(\sigma_{\theta \theta}^{I I}\right)_{h}^{*}$ and $\left(\sigma_{r \theta}^{I I}\right)_{h}^{*}$ are exactly the same as Appendix A except changing $a(v h-1) \sin \alpha$ in $\Psi_{a}$ and $\Psi_{b}$ to $b(v h-1) \sin \alpha$, that is

$\Psi_{a}=\left[h^{2}-a^{2}(v h-1)^{2}\right]^{1 / 2}\left[\Theta_{a}-b(v h\right.$

$-1) \sin \alpha]\left[\Theta_{a}-c(v h-1)\right] S_{-}^{o}\left(\Theta_{a} /(v h-1)\right)$,

$\Psi_{b}=\left[h^{2}-b^{2}(v h-1)^{2}\right]^{1 / 2}\left[\Theta_{b}-b(v h\right.$

$-1) \sin \alpha]\left[\Theta_{b}-c(v h-1)\right] S_{-}^{o}\left(\Theta_{b} /(v h-1)\right)$. 